in remote places, where the only information on cancer prevalence is that derived from the persons who happen to find their way into hospitals and into cancer registry files.

At present the I.A.R.C. is undertaking studies in areas where cancer of the oesophagus is prevalent. In this connexion the Northern Gonbad region of Iran is of special interest because, unlike other high-incidence areas, the rate in women is more than that for men. In that region there is so far no clue to the nature of the causative factor. In Brittany, on the other hand, a high incidence of the disease in men seems to be associated with a high incidence of both alcoholism and cirrhosis of the liver.

An interesting part of the I.A.R.C.'s programme is a study designed to see whether data from cancer registries could in future be used to give early warning of the introduction of new cancer hazards into the environment. At the same time, in collaboration with the Division of Health Statistics of the World Health Organization, the I.A.R.C. is examining the need for revision of the cancer section of the Internanational Classification of Diseases, and experimental studies on polycyclic aromatic hydrocarbons, nitrosamines, aflatoxin, and asbestos have been organized on a collaborative basis.

Despite its need for a larger budget and the slow speed at which its permanent buildings seem to be going up, the International Agency has already succeeded in having an impact in several important aspects of research on a disease which year by year grows in relative importance as the risk of premature death from other causes recedes.

${ }^{1}$ British Medical fournal, 1965, 1, 1327.

2 British Medical fournal, 1965, 1, 1327

3 International Agency for Research on Cancer, Annual Report 1970. Lyon, 1971.

\section{Surgery of Patent Ductus Arteriosus}

Over 30 years ago R. E. Gross and J. P. Hubbard reported the first successful ligation of a patent ductus arteriosus. ${ }^{1}$ Since then a wide experience of the operation has been gained in many centres all over the world, and a recent article from the team at the Hospital for Sick Children in London has reviewed their results in a total of 936 procedures carried out between 1946 and 1969.2

The rationale for operating on a patent ductus is provided by the natural history of the lesion. ${ }^{3} \mathrm{M}$. Campbell has calculated that only $0.6 \%$ of all persistent ductus would close spontaneously every year. Moreover, the death rate is heavy in the first two years of life, though thereafter only $0.5 \%$ die each year in the first two decades. Nevertheless, by the age of 10 years the risk of bacterial endocarditis increases to $0.45 \%$ per annum and this continues for 40 years or more. At the beginning of the third decade the death rate increases from heart failure due to various causes associated with the persistent duct and reaches $20 \%$ by the age of 30. Further complications which develop in older adults are calcification of the ductus, aneurysmal dilatation of the aortic end, pulmonary hypertension, left ventricular hypertrophy, and heart failure. Thus by the age of 60 roughly $60 \%$ of untreated patients will have died. Since in uncomplicated cases closure of the ductus is a safe surgical procedure, most children now have this operation performed, and further figures relating to unoperated ducts are unlikely ever to be produced.
There are two surgical methods of dealing with patent ductus-namely, ligation and division-and there has been much debate about which is better. Some workers in the United States have tended to favour division, ${ }^{4}$ but undoubtedly in the uncomplicated case ligation is adequate. Division is reserved for large ducts where ligation might be risky, and it carries a higher morbidity, though it eliminates any risk of recanalization. In the vast majority of the series described from the Hospital for Sick Children the treatment was by ligation using two ligatures of thick plaited silk, and the results were excellent.

Of the 936 consecutive cases closed surgically in infants and children, there were 789 without any other cardiac anomaly. In this group there were 11 deaths, but seven of these had severe congenital anomalies other than cardiovascular. Most of the deaths in this series, as would be expected, were associated with additional congenital cardiac lesions, 48 out of 197 . Of these deaths, 47 occurred in infants, and of 691 patients over the age of 1 year the hospital mortality rate was less than $0.5 \%$. The commonest additional heart lesions were coarctation and ventricular septal defect, but other lesions such as aortic and mitral valve disease, pulmonary stenosis, and transposition of the great arteries also occurred as well as several highly complicated congenital lesions.

The authors confirm that a patent ductus presenting the classical clinical signs and diagnosed by a cardiologist as a solitary lesion does not need further investigation, and many of their cases were referred straight for surgery on clinical diagnosis alone. The development or persistence of pulmonary hypertension with an increased pulmonary vascular resistance is the main worry about delay in treating patent ductus arteriosus. Eventually, irreversible changes will ensue and operation will no longer be feasible. In this series pulmonary hypertension occurred in 28 patients with an isolated patent ductus and in another 38 patients with additional cardiac anomalies. When the pulmonary vascular resistance was close to the systemic vascular resistance, the pressures in both arterial systems were recorded at operation, and ligation was not proceeded with unless the pulmonary artery pressure fell after trial occlusion of the ductus. In five patients in this series the pressure was found to increase or remained unchanged and so operation was not carried out. Of the 66 patients known to have pulmonary hypertension, 10 died in hospital, and despite operation a further three died some years later from severe and increasing pulmonary vascular disease.

If the patent ductus had resulted in heart failure, enlargement of the heart, or pulmonary hypertension, operation was performed as soon as possible after diagnosis whatever the age. Generally, however, the age regarded as optimal for operation varies from unit to unit and in most places the operation is postponed until the child is walking and can feed itself. Ideally, the operation should be completed before the child starts school and the team from the Hospital for Sick Children recommend 4 to 5 years as the most suitable age.

Of the postoperative complications recanalization of the ductus is the one most widely recognized, though this is now extremely rare; in this series the complication occurred in only four children and in no case was it fatal.

In summary, therefore, this very large series presents unequivocal evidence that ligation of the patent ductus arteriosus in a child without other associated lesions is a safe and curative operation. It should be carried out before the age of 5 so that the child can grow up normally with 
none of the consequences of leaving the duotus unclosed. The cases of patent ductus arteriosus with associated lesions are very different, and the latter will determine the prognosis and the management. This is the group which present principally in infancy, the mortality is much higher, and surgical treatment may be a matter of urgency.

${ }^{1}$ Gross, R. E., and Hubbard, J. P., Fournal of the American Medical Association, 1939, 112, 729 .

Panagopoulos, P. G., Tatodes, C. J., Aberdeen, E., Waterston, D. J., and Carter, R. E. B., Thorax, 1971, 26, 137.

Campbell, M., British Heart fournal, 1968, 30, 1, 4.

- Gross, R. E., Fournal of Thoracic Surgery, 1947, 16, 314.

\section{Nobel Prize}

The history of biological research is punctuated by discoveries whose implications have changed the course of medical scientific thought. Such events as the discovery of insulin and the unravelling of the intricacies of protein structure have had far-reaching consequences. Into this category falls the work of Earl Sutherland, professor of physiology at the Vanderbilt University School of Medicine, Nashville, Tennessee, whose award of the 1971 Nobel prize for physiology or medicine was announced last week.

In the 1940s Sutherland began a series of experiments on the mechanism by which adrenaline and glucagon stimulated the conversion of glycogen to glucose in the liver. $\mathrm{He}$ found that these hormones increased the amount of an enzyme, phosphorylase, within the liver, by an indirect and complicated mechanism. Up to 1956 he was able to show this function of adrenaline only in intact liver cells. But in 1957 T. W. Rall, E. W. Sutherland, and J. Berthet ${ }^{1}$ showed that these hormones could activate the enzyme in a cell-free system-namely, a liver homogenate. Further work by Sutherland and his colleagues showed that merely by centrifuging the liver homogenate it was possible to deplete the system of the enzyme activity under study, and addition of the hormones to the supernatant portion produced after centrifugation failed to reactivate it. However, when the hormones were added to the cellular debris of centrifugation they stimulated the formation of a heat-stable factor that did activate the enzymes. This factor was isolated and later identified by the Nashville workers as adenosine $3^{\prime}, 5^{\prime}$-monophosphate, better known as cyclic AMP. Cyclic AMP is formed from adenosine triphosphate (ATP) by an enzyme, adenyl cyclase, which occurs in cell membranes.

After this discovery Sutherland went on to formulate a concept that has won increasing recognition over the years -namely, that cyclic AMP is an essential link in the mode of action of many, if not most, hormones and many drugs. According to his ideas hormones (or first messengers) travel from their tissue of origin to their target organ, where by changing the intracellular concentration of a second messenger-namely, cyclic AMPchanges specific to the first messenger are produced in the tissues. Among hormones whose action is probably mediated by cyclic AMP are catecholamines (affecting both glucose and fat metabolism), glucagon, ACTH, vasopressin, follicle-stimulating hormone, parathyroid hormone, calcitonin, insulin, prostaglandins, and several others. At least a dozen hormones either raise the intracellular cyclic AMP concentration or lower it, and one mechanism whereby hormone antagonism may occur is by exerting opposite effects on cyclic AMP. Drugs whose action depends on the adenyl-cyclase/cyclic-AMP system include beta stimulants such as isoprenaline and beta blockers such as propranolol. Others drugs such as caffeine and theophylline slow the rate of destruction of cyclic AMP and thus may potentiate hormonal action.

The precise molecular mode of action of cyclic AMP is only partly unravelled, but its overall role in the control of many physiological processes is now clear. Its relation to disease in man remains to be explored. Since many hormones act through this messenger, it is reasonable to suppose that diseases characterized by hormone overactivity may be associated with increased tissue levels of cyclic AMP and that states of deficiency of hormones would be associated with low tissue levels. Urinary levels of cyclic AMP in patients with parathyroid disease in fact follow this pattern. The fascinating problem of tumours with functional hormonal effects may represent another facet of the cyclic AMP story, and perhaps congenital deficiencies of hormones are due to malfunction of the second messenger system.

The biological implications of Sutherland's work are immense. So far they have largely involved physiologists, biochemists, and pharmocologists, but it is clear that the ripples are fast spreading to the frontiers of clinical medicine and human disease.

\footnotetext{
1 Rall, T. W., Sutherland, E. W., Berthet, J. Fournal of Biological
} Chemistry, 1957 224, 463. 